\title{
MicroRNAs, mRNAs, and Translation
}

\author{
P.A. Maroney, Y. Yu, AND T.W. Nilsen \\ Center for RNA Molecular Biology and Department of Biochemistry, Case Western Reserve University \\ School of Medicine, Cleveland, Ohio 44106-4973
}

\begin{abstract}
MicroRNAs (miRNAs) comprise a large family of regulatory molecules that repress protein production from targeted mRNAs. Although it is now clear that miRNAs exert pervasive effects on gene expression in animal cells, the mechanism(s) by which they function remains poorly understood. We have analyzed the subcellular distribution of miRNAs in actively growing HeLa cells and find that the vast majority are associated with actively translating mRNAs in polysomes. We also find that a specific miRNA-regulated mRNA (KRAS) is polysome associated and that its translation is impaired, apparently at the level of elongation. These observations are discussed in light of our current understanding of mechanism of miRNA function.
\end{abstract}

MicroRNAs (miRNAs), initially discovered in Caenorhabditis elegans as posttranscriptional regulators of genes involved in developmental timing, are now known to regulate the expression of the majority of genes in animals (Ambros 2004; Bartel 2004; Farh et al. 2005; Stark et al. 2005). Several hundred miRNAs have been characterized, and each miRNA has several hundred targets. Compelling informatic and experimental evidence indicates that miRNAs primarily recognize their targets via limited Watson-Crick base-pairing interactions between the $5^{\prime}$ end of the miRNA (nucleotides 2-8) and the 3'UTRs. Like other functional noncoding RNAs, miRNAs do not act as naked RNAs but rather exert their effects as ribonucleoproteins (RNPs); a common constituent of all miRNPs is a member of the argonaute (AGO) protein family (for review, see Bartel 2004; Pillai 2005). Although it is widely accepted that miRNA binding to a target mRNA is sufficient to elicit repression of protein production from that $\mathrm{mRNA}$, there is at present no consensus as to how down-regulation takes place. Indeed, there is evidence for multiple distinct mechanisms including translational repression, enhanced mRNA degradation, or sequestration in cytoplasmic foci known as processing bodies (P-bodies). How or whether these different mechanisms are related is not yet clear. Here, we discuss recent results from our laboratory in the context of the current understanding of mechanisms of regulation by miRNAs and highlight what appear to be open questions.

\section{MECHANISMS OF REPRESSION OF GENE EXPRESSION BY MIRNAS}

The earliest analyses of miRNA mechanism of action were done in C. elegans, and it was found that miRNAtargeted mRNAs were present when no protein encoded by those mRNAs could be detected. Moreover, the targeted mRNAs appeared to be associated with ribosomes in polysomes (Olsen and Ambros 1999; Seggerson et al. 2002). These results suggested that the block to synthesis of protein was after initiation of protein synthesis either by a direct effect on translation elongation or cotransla- tional degradation of the newly synthesized proteins. As discussed below, these early results have not yet been explained. In subsequent analyses, it appears that much of the regulation of these initially recognized miRNA targets might be due to the degradation of the targeted mRNAs (Bagga et al. 2005), but the reasons for the discrepancies between studies conducted at different times are not yet clear. Regardless, there are now abundant examples where miRNA-mediated regulation represses protein production but does not result in lowered mRNA levels (see, e.g., O’Donnell et al. 2005).

Just as clearly, there is a significant and expanding literature which demonstrates that miRNAs can destabilize certain targeted mRNAs (Bagga et al. 2005; Lim et al. 2005; Behm-Ansmant et al. 2006; Giraldez et al. 2006; Rehwinkel et al. 2006). The fact that miRNA-mRNA interaction can result in enhanced turnover has enabled the use of mRNA microarray analysis to identify targeted miRNAs. A major question in the field is what fraction of total targets are identified by these types of experiments. There exists no analogous method to identify targets that are regulated strictly at the translational level. Furthermore, there is no straightforward way to determine whether the level of mRNA reduction corresponds to the total level of regulation; i.e., some mRNAs may be regulated both by destabilization and at the level of translation. A recent report highlights these issues; here, regulation of specific reporter constructs was observed to be mediated exclusively at the level of translation, a combination of translation and stability, or stability alone (Behm-Ansmant et al. 2006). A major challenge will be to determine how specific mRNAs are targeted for different modes of regulation.

The fact that specific regulated mRNAs appear to be regulated by distinct mechanisms is further complicated by data which suggest that each "mechanism," i.e., translational repression, or destabilization might be idiosyncratic for specific mRNAs. In this regard, there is evidence that translational repression can occur at several different steps. Two studies have demonstrated miRNAmediated inhibition of translation at the level of initiation 
(Humphreys et al. 2005; Pillai et al. 2005), and a third has provided evidence that initiation is not affected, but rather that translating ribosomes disengage prematurely (drop off) from a miRNA-regulated mRNA (Petersen et al. 2006). Although different approaches were used in these studies, it is difficult at present to rationalize the disparity in the results.

Our understanding of destabilization is somewhat clearer, but even here there are several unanswered questions. Three studies have shown that miRNA-mRNA interaction can cause deadenylation of targeted mRNAs (Behm-Ansmant et al. 2006; Giraldez et al. 2006; Wu et al. 2006). In some cases, this deadenylation leads to mRNA degradation via subsequent decapping (BehmAnsmant et al. 2006), but in other cases, it does not. Furthermore, even in the cases where deadenylation does not lead to instability, it alone does not account for the level of translational inhibition (Behm-Ansmant et al. 2006; Wu et al. 2006).

Recently, there has been considerable interest and excitement over the potential role of processing bodies (P-bodies), also known as GW-182 bodies, in miRNA function. Originally described in budding yeast, P-bodies are cytoplasmic foci that contain a variety of factors including decapping enzymes, deadenylases, and exonucleases known to be involved in and required for mRNA decay (for review, see Valencia-Sanchez et al. 2006). In yeast, these foci can serve as a repository for untranslated mRNAs that can either be degraded or return to the translating pool (Brengues et al. 2005). The demonstration by several groups that Ago 2 interacts (directly or indirectly) with decapping enzymes led to the finding that Ago 2 localizes to these foci (Liu et al. 2005a,b; Sen and Blau 2005). A number of other provocative studies have demonstrated miRNA-dependent localization of targeted mRNAs to P-bodies (Liu et al. 2005a) and have established a role for the P-body constituent GW-182 in miRNA function (Jakymiw et al. 2005; Liu et al. 2005b; Rehwinkel et al. 2005; Behm-Ansmant et al. 2006).

Collectively, these analyses have led to the attractive hypothesis that P-bodies are central to miRNA function (for review, see Valencia-Sanchez et al. 2006). This hypothesis has the advantage of rationalizing many, but not all (see below), of the disparate observations pertaining to miRNA action. In this regard, the apparent diversity of mechanisms could result from different fates of mRNAs sequestered in P-bodies; i.e., some could be destined for decay while others could be held in stasis spatially removed from the translational machinery (P-bodies are devoid of ribosomes) (for review, see ValenciaSanchez et al. 2006).

Although the P-body hypothesis is appealing, there are several open questions. First, what fraction of the total cellular complement of P-body-associated proteins (e.g., Ago 2, GW-182, etc.) is actually present in microscopically visible foci? Studies to date have not been quantitative. Second, is the "structural integrity" of P-bodies important for miRNA-mediated gene regulation? This has been a difficult question to address because P-bodies have resisted attempts at biochemical purification and, therefore, a definition of what constitutes a P-body has not yet emerged. Notably, however, a recent study has shown that depletion of an LSm protein eliminates visualizable P-bodies but does not impair miRNA function (Chu and Rana 2006). Whether submicroscopic foci remain under these conditions is not clear. Third, what fraction of translationally repressed mRNAs are concentrated in P-bodies and therefore disengaged from ribosomes?

With the exception of mRNA microarray studies, which can only monitor mRNA levels, most mechanistic analyses of miRNA function have focused on specific miRNA-mRNA combinations; reporter constructs responsive to endogenous or "designer" miRNAs. To gain a different perspective, we thought that it would be informative to analyze the subcellular partitioning of bulk miRNAs. We anticipated that the "behavior" of endogenous miRNAs would be diagnostic for their mode(s) of regulation. As detailed below, these studies yielded unexpected results.

\section{MOST MIRNAS IN ACTIVELY GROWING HELA CELLS ARE ASSOCIATED WITH TRANSLATING MRNAS}

To analyze the subcellular partitioning of bulk miRNAs in HeLa cells, we prepared cytoplasmic extracts from exponentially growing cells and fractionated them on sucrose gradients. When individual fractions were analyzed for the presence of three abundant miRNAs, we were surprised to find that the vast majority co-sedimented with polysomes. By comparing the amounts of miRNAs recovered in the gradients to total miRNAs present in the cell, it was clear that most of the miRNAs were indeed present in the cytoplasmic extracts and most cofractionated with polysomes; i.e., very few miRNAs were pelleted.

Several experiments, including dissociation of the ribosomes with EDTA, indicated that the miRNAs were polysome associated and not simply co-sedimenting with polysomes. Importantly, mild digestion of the extracts with micrococcal nuclease, which digests mRNAs in polysomes but leaves ribosomes intact, released the miRNAs such that they migrated at the top of the gradient. This experiment strongly suggested that the miRNAs were associated with polysomes because they were bound to mRNAs.

To determine whether the mRNAs associated with miRNAs were being translated, cells were treated under a variety of conditions that affect bulk protein synthesis. When cells were treated with pactamycin, which at low concentrations predominately inhibits initiation of translation (Taber et al. 1971), we observed significant accumulation of $80 \mathrm{~S}$ ribosomes, as expected. When initiation is disrupted, elongating ribosomes finish translation and dissociate from the mRNAs. Concomitant with the inhibition of protein synthesis, mRNAs shift from heavy polysomes to lighter ones reflecting runoff of elongating ribosomes. Remarkably, the same shift from heavy to light ribosomes was observed for the miRNAs. Similarly, when cells were treated with puromycin, a drug that inhibits protein synthesis by causing premature termination of translation, similar results were obtained; i.e., 
mRNAs and miRNAs shifted from heavy polysomes to lighter ones. With both pactamycin and puromycin treatment, it was clear that miRNA sedimentation paralleled that of mRNAs, not bulk ribosomal RNA, thus providing additional evidence that the miRNAs we analyzed were associated with mRNAs. Furthermore, these experiments indicated that most miRNAs were associated with mRNAs that were being actively translated.

Both pactamycin and puromycin are irreversible inhibitors of protein synthesis. We wished to assess the effect of miRNA-mRNA interaction under conditions where protein synthesis could be arrested and then allowed to resume. To do this, we treated cells with hypertonic media. Hypertonic shock rapidly arrests protein synthesis by inhibiting initiation and, importantly, protein synthesis recovers upon return to isotonic media (Morley and Naegele 2002). As expected from the pactamycin results, inhibition of initiation by hypertonic shock caused disaggregation of polysomes due to ribosome runoff and a large accumulation of $80 \mathrm{~S}$ monosomes. As a consequence of this inhibition of protein synthesis, both mRNAs and miRNAs shifted in sedimentation to lighter fractions. When cells were returned to isotonic media, there was a rapid recovery of protein synthesis and both mRNAs and miRNAs returned to heavy polysomal fractions. These results clearly indicate that most miRNA-mRNA interactions are compatible with protein synthesis and do not interfere with resumption of protein synthesis after it is arrested.

How can one interpret these results in light of other studies? First, the finding that most miRNAs are associated with polysomes may not be as surprising as it appears at first glance. In this regard, the first identified miRNAtargeted mRNAs in C. elegans were demonstrated to be polysome associated (Olsen and Ambros 1999; Seggerson et al. 2002), and subsequent studies have shown that the bulk of miRNAs in Drosophila and C. elegans were present in ribosomal fractions (Hammond et al. 2000, 2001; Caudy et al. 2003). Furthermore, there have been several reports of miRNAs present in polysomes (see, e.g., Kim et al. 2004; Nelson et al. 2004), and in one case, miRNA-target mRNA interaction in polysomes was documented (Nelson et al. 2004).

We believe that it is most likely that the miRNA-mRNA interactions we observe are mediated by base-pairing between the miRNAs and target mRNAs and thus are "active," but this is technically impossible to prove. Nevertheless, if we assume that the polysomeassociated miRNAs are functioning as regulators, what are the implications of our results regarding mechanism? It would seem that they are in conflict with the P-body hypothesis described above, since we do not observe any accumulation of non-polyribosome-associated miRNAs. However, this may not be the case. It is possible that regulated mRNAs could exit translation and be degraded or sequestered in a P-body-component-dependent manner that involves the release of the regulatory miRNA. If this were the case, no steady-state accumulation of miRNAs in P-bodies would be expected. In this scenario, the apparent localization of Ago 2 to P-bodies (Liu et al. 2005a; Sen and Blau 2005) would be the result of tran- sient interactions. Clearly, a quantitative assessment of the fraction of Ago 2 present in P-bodies would be helpful in determining whether this conjecture is correct. It would also be informative to determine the complement of intact mRNAs present in P-bodies and whether they are associated with miRNAs, but such analyses await the development of techniques to isolate these entities.

Regardless of the role(s) of P-body components in miRNA-mediated regulation, our results clearly indicate that miRNA-mRNA interactions are not necessarily dramatically repressive to protein synthesis. They are therefore consistent with a large number of studies which indicate that the majority of mRNAs in animal cells are miRNA targets but are still translated (see, e.g., Farh et al. 2005). Indeed, with few exceptions, the magnitude of regulation by miRNAs appears to be quite modest (see, e.g., Lewis et al. 2003; Lim et al. 2005; and see also Bartel and Chen 2004). In principle, degrees of regulation could be achieved by regulating accessibility of the miRNA to its targets. However, there is no evidence for compartmentalization of miRNAs and targets, and our data together with that of others (Kim et al. 2004; Nelson et al. 2004) suggest that regulation is primarily achieved at the level of the actively translating ribosome mRNA complex. This view is consistent with the recent demonstration that miRNA function requires the participation of Rck/p54 (Chu and Rana 2006), a DEAD box RNA helicase, whose homolog in yeast, Dhh-1, is required for translational inhibition (Coller and Parker 2005). Notably, Dhh-1 only exerts its effects on mRNAs that have engaged the translational machinery (Coller and Parker 2005).

\section{A SPECIFIC MIRNA-REGULATED MRNA IS PRESENT IN POLYSOMES AND TRANSLATIONALLY IMPAIRED}

Although the experiments described above were informative regarding the behavior of bulk miRNAs, we wanted to determine how a specific miRNA-regulated mRNA partitioned within the cell. For these analyses we chose the KRAS mRNA because it has been shown to be regulated by the let-7 miRNA in HeLa cells (Johnson et al. 2005; Chu and Rana 2006). When the subcellular distribution of this mRNA was examined, it was found to cosediment with polysomes, and sedimentation was sensitive to treatment with EDTA, as expected if it were ribosome associated. More importantly, unlike the behavior of control mRNAs, the sedimentation of KRAS demonstrated reduced sensitivity to puromycin treatment and reduced sensitivity to a block to initiation of protein synthesis caused by hypertonic shock. These observations strongly suggest that miRNA-mediated inhibition of KRAS protein syntheses is due to impairment of translation elongation.

Our observations with KRAS mRNA clearly are not consistent with a block to initiation of protein synthesis (Humphreys et al. 2005; Pillai et al. 2005); if this were the case, the mRNA would not be polysome associated. They also are not compatible with enhanced rate of ribosome dropoff post-initiation (Petersen et al. 2006); if this were the case, we would have observed changes in sedimenta- 
tion upon exposure to hypertonic conditions. They are also not compatible with sequestration in P-bodies because these structures lack ribosomes (ValenciaSanchez et al. 2006). Although the behavior of KRAS does not fit well with current models of miRNA-mediated translational down-regulation, it appears to be strikingly similar to the original miRNA targets described in C. elegans (Olsen and Ambros 1999; Seggerson et al. 2002) which were found to be polysome associated. The behavior of KRAS mRNA also appears to be similar to that of the pal-1 mRNA in C. elegans which is translationally down-regulated by Gld-1. In that case, several lines of evidence suggest that translation is inhibited by the slowing or stalling of elongating ribosomes (Mootz et al. 2004). Other examples of post-initiation repression exist (Clark et al. 2000; Braat et al. 2004); whether these are miRNA-mediated is not known. It will be of considerable interest to determine whether apparently disparate cases of regulation at the level of elongation require common factors; e.g., GW182.

Our finding that KRAS is apparently down-regulated at the level of translation elongation is, as noted above, consistent with the earliest analyses of mechanism of miRNA function and it raises the possibility that other polysomeassociated miRNAs also affect elongation but to a lesser extent. In this regard, a modest slowing of translation would be invisible in our analysis of bulk miRNAs.

\section{CONCLUSIONS}

It is now apparent that miRNAs can regulate protein production by targeted mRNAs in multiple ways and to widely varying extents. Given that only a few examples have been studied in any detail, it is not yet clear that all possible mechanisms have been uncovered. Furthermore, with the possible exception of enhanced turnover, the molecular details of how repression occurs are not known.

It will be important to determine how many and which mRNAs are regulated at the level of translation alone, stability, or combination of both. This will be difficult, since no technique currently allows monitoring of translation on the scale of mRNA microarray analyses. However, it may be possible to use this type of experiment to identify mRNAs that remain in polysomes when translation initiation is arrested; i.e., mRNAs highly regulated at the level of translation elongation.

Equally challenging will be defining the factors that determine the magnitude of regulation that occurs by any given mechanism. In this regard, it seems unlikely that different modes or magnitudes of regulation are specified by the miRNAs themselves. Although many potential "auxiliary" factors have been identified, it does not appear that they function in an mRNA-specific fashion (see, e.g., Caudy et al. 2002; Mourelatos et al. 2002; Meister et al. 2005). Furthermore, studies that have revealed inhibition of protein synthesis at the level of initiation (Pillai et al. 2005) and elongation (see above) used distinct 3'UTRs responsive to the same miRNA, let-7. In addition, there are numerous examples of differences in magnitude of regulation elicited by the same miRNA (see, e.g., Lewis et al. 2003; Lim et al. 2005). It thus appears that both mechanism and magnitude of regulation are determined by the 3'UTR context in which the miRNA binds to the target mRNA. Indeed, recent analyses of a specific miRNA target in C. elegans provide a clear illustration of dramatic context effects (Didiano and Hobert 2006). Presumably, these effects are mediated by the presence of distinct sets of proteins bound to different 3'UTRs. Elucidating the identity of such proteins and how they interact with the miRNA regulatory machinery will clearly be important in understanding the overall impact of miRNAs on gene expression.

\section{ACKNOWLEDGMENTS}

The described work from our laboratory is currently in press in Nature Structural and Molecular Biology and was supported by grants from the National Institutes of Health.

\section{REFERENCES}

Ambros V. 2004. The functions of animal microRNAs. Nature 431: 350 .

Bagga S., Bracht J., Hunter S., Massirer K., Holtz J., Eachus R., and Pasquinelli A.E. 2005. Regulation by let-7 and lin-4 miRNAs results in target mRNA degradation. Cell 122: 553.

Bartel D.P. 2004. MicroRNAs: Genomics, biogenesis, mechanism, and function. Cell 116: 281.

Bartel D.P. and Chen C.-Z. 2004. Micromanagers of gene expression: The potentially widespread influence of metazoan microRNAs. Nat. Rev. Genet. 5: 396.

Behm-Ansmant I., Rehwinkel J., Doerks T., Stark A., Bork P., and Izaurralde E. 2006. mRNA degradation by miRNAs and GW182 requires both CCR4:NOT deadenylase and DCP1:DCP2 decapping complexes. Genes Dev. 20: 1885.

Braat A.K., Yan N., Arn E., Harrison D., and Macdonald P.M. 2004. Localization-dependent oskar protein accumulation: Control after the initiation of translation. Dev. Cell 7: 125.

Brengues M., Teixeira D., and Parker R. 2005. Movement of eukaryotic mRNAs between polysomes and cytoplasmic processing bodies. Science 310: 486 .

Caudy A.A., Myers M., Hannon G.J., and Hammond S.M. 2002. Fragile X-related protein and VIG associate with the RNA interference machinery. Genes Dev. 16: 2491.

Caudy A.A., Ketting R.F., Hammond S.M., Denli A.M., Bathoorn A.M., Tops B.B., Silva J.M., Myers M.M., Hannon G.J., and Plasterk R.H. 2003. A micrococcal nuclease homologue in RNAi effector complexes. Nature 425: 411.

Chu C.-Y. and Rana T.M. 2006. Translation repression in human cells by MicroRNA-induced gene silencing requires RCK/p54. PLoS Biol. 4: e210.

Clark I.E., Wyckoff D., and Gavis E.R. 2000. Synthesis of the posterior determinant Nanos is spatially restricted by a novel cotranslational regulatory mechanism. Curr. Biol. 10: 1311.

Coller J. and Parker R. 2005. General translational repression by activators of mRNA decapping. Cell 122: 875.

Didiano D. and Hobert O. 2006. Perfect seed pairing is not a generally reliable predictor for miRNA-target interactions. Nat. Struct. Mol. Biol. 13: 849.

Farh K.K., Grimso A., Jan C., Lewis B.P., Johnston W.K., Lim L.P., Burge C.B., and Bartel D.P. 2005. The widespread impact of mammalian MicroRNAs on mRNA repression and evolution. Science 310: 1817.

Giraldez A.J., Mishima Y., Rihel J., Grocock R.J., Van Dongen S., Inoue K., Enright A.J., and Schier A.F. 2006. Zebrafish MiR-430 promotes deadenylation and clearance of maternal mRNAs. Science 312: 75.

Hammond S.M., Bernstein E., Beach D., and Hannon G.J. 2000. An RNA-directed nuclease mediates post-transcriptional gene silencing in Drosophila cells. Nature 404: 293. 
Hammond S.M., Boettcher S., Caudy A.A., Kobayashi R., and Hannon G.J. 2001. Argonaute2, a link between genetic and biochemical analyses of RNAi. Science 293: 1146.

Humphreys D.T., Westman B.J., Martin D.I., and Preiss T. 2005. MicroRNAs control translation initiation by inhibiting eukaryotic initiation factor 4E/cap and poly(A) tail function. Proc. Natl. Acad. Sci. 102: 16961.

Jakymiw A., Lian S., Eystathioy T., Li S., Satoh M., Hamel J.C., Fritzler M.J., and Chan E.K. 2005. Disruption of GW bodies impairs mammalian RNA interference. Nat. Cell Biol. 7: 1267.

Johnson S.M., Grosshans H., Shingara J., Byrom M., Jarvis R., Cheng A., Labourier E., Reinert K.L., Brown D., and Slack F.J. 2005. RAS is regulated by the let-7 microRNA family. Cell 120: 635

Kim J., Krichevsky A.M., Grad Y., Hayes G.D., Kosik K.S., Church G.M., and Ruvkun G. 2004. Identification of many microRNAs that copurify with polyribosomes in mammalian neurons. Proc. Natl. Acad. Sci. 101: 360.

Lewis B.P., Shih I.-H., Jones-Rhoades M.W., Bartel D.P., and Burge C.B. 2003. Prediction of mammalian microRNA targets. Cell 115: 787.

Lim L.P., Lau N.C., Garrett-Engele P., Grimson A., Schelter J.M., Castle J., Bartel D.P., Linsley P.S., and Johnson J.M. 2005 Microarray analysis shows that some microRNAs downregulate large numbers of target mRNAs. Nature 433: 769.

Liu J., Valencia-Sanchez M.A., Hannon G.J., and Parker R. 2005a. MicroRNA-dependent localization of targeted mRNAs to mammalian P-bodies. Nat. Cell Biol. 7: 719.

Liu J., Rivas F.V., Wohlschlegel J., Yates J.R., III, Parker R., and Hannon G.J. 2005b. A role for the P-body component GW182 in microRNA function. Nat. Cell Biol. 7: 1261.

Meister G., Landthaler M., Peters L., Chen P.Y., Urlaub H., Luhrmann R., and Tuschl T. 2005. Identification of novel argonaute-associated proteins. Curr. Biol. 15: 2149

Mootz D., Ho D.M., and Hunter C.P. 2004. The STAR/Maxi$\mathrm{KH}$ domain protein GLD-1 mediates a developmental switch in the translational control of C. elegans PAL-1. Development 131: 3263.

Morley S.J. and Naegele S. 2002. Phosphorylation of eukaryotic initiation factor (eIF) 4E is not required for de novo protein synthesis following recovery from hypertonic stress in human kidney cells. J. Biol. Chem. 277: 32855.

Mourelatos Z., Dostie J., Paushkin S., Sharma A., Charroux B., Abel L., Rappsilber J., Mann M., and Dreyfuss G. 2002. miRNPs: A novel class of ribonucleoproteins containing numerous microRNAs. Genes Dev. 16: 720.

Nelson P.T., Hatzigeorgiou A.G., and Mourelatos Z. 2004. miRNP:mRNA association in polyribosomes in a human neuronal cell line. RNA 10: 387 .

O'Donnell K.A., Wentzel E.A., Zeller K.I., Dang C.V., and Mendell J.T. 2005. c-Myc-regulated microRNAs modulate E2F1 expression. Nature 435: 839.

Olsen P.H. and Ambros V. 1999. The lin-4 regulatory RNA controls developmental timing in Caenorhabditis elegans by blocking LIN-14 protein synthesis after the initiation of translation. Dev. Biol. 216: 671

Petersen C.P., Bordeleau M.E., Pelletier J., and Sharp P.A. 2006. Short RNAs repress translation after initiation in mammalian cells. Mol. Cell 21: 533.

Pillai R.S. 2005. MicroRNA function: Multiple mechanisms for a tiny RNA? RNA 11: 1753

Pillai R.S., Bhattacharyya S.N., Artus C.G., Zoller T., Cougot N., Basyuk E., Bertrand E., and Filipowicz W. 2005. Inhibition of translational initiation by let-7 microRNA in human cells. Science 309: 1573.

Rehwinkel J., Behm-Ansmant I., Gatfield D., and Izaurralde E. 2005. A crucial role for GW182 and the DCP1:DCP2 decapping complex in miRNA-mediated gene silencing. RNA 11: 1640 .

Rehwinkel J., Natalin P., Stark A., Brennecke J., Cohen S.M., and Izaurralde E. 2006. Genome-wide analysis of mRNAs regulated by Drosha and Argonaute proteins in Drosophila melanogaster. Mol. Cell. Biol. 26: 2965.

Seggerson K., Tang L., and Moss E.G. 2002. Two genetic circuits repress the Caenorhabditis elegans heterochronic gene lin-28 after translation initiation. Dev. Biol. 243: 215.

Sen G.L. and Blau H.M. 2005. Argonaute 2/RISC resides in sites of mammalian mRNA decay known as cytoplasmic bodies. Nat. Cell Biol. 7: 633-636.

Stark A., Brennecke J., Bushati N., Russell R.B., and Cohen S.M. 2005. Animal microRNAs confer robustness to gene expression and have a significant impact on 3'UTR evolution. Cell 123: 1133.

Taber R., Rekosh D., and Baltimore D. 1971. Effect of pactamycin on synthesis of poliovirus proteins: A method for genetic mapping. J. Virol. 8: 395.

Valencia-Sanchez M.A., Liu J., Hannon G.J., and Parker R. 2006. Control of translation and mRNA degradation by miRNAs and siRNAs. Genes Dev. 20: 515.

Wu L., Fan J., and Belasco J.G. 2006. MicroRNAs direct rapid deadenylation of mRNA. Proc. Natl. Acad. Sci. 103: 4034. 


\section{$8_{\mathrm{CSH}}^{\infty}$ Cold Spring Harbor Symposia SYMPOSIA}

\section{MicroRNAs, mRNAs, and Translation}

P.A. MARONEY, Y. YU and T.W. NILSEN

Cold Spring Harb Symp Quant Biol 2006 71: 531-535

Access the most recent version at doi:10.1101/sqb.2006.71.043

References This article cites 43 articles, 19 of which can be accessed free at: http://symposium.cshlp.org/content/71/531.full.html\#ref-list-1

\section{License}

Email Alerting Receive free email alerts when new articles cite this article - sign up in Service the box at the top right corner of the article or click here. 